\title{
The iNAPO Project: Biomimetic Nanopores for a New Generation of Lab-on-Chip Micro Sensors
}

\author{
Wolfgang Ensinger¹, Mubarak Ali, ${ }^{1,2}$, Saima Nasir'1, Ivana Duznovic ${ }^{1}$, Christina Trautmann ${ }^{1,2}$, \\ Maria Eugenia Toimil-Molares ${ }^{2}$, Giuseppa R. Distefano ${ }^{1,2}$, Bodo Laube ${ }^{1}$, Max Bernhard ${ }^{1}$, \\ Melanie Mikosch-Wersching ${ }^{1}$, Helmut F. Schlaak ${ }^{1}$, Mario El Khoury ${ }^{1}$ \\ ${ }^{1}$ Technische Universität Darmstadt \\ Karolinenplatz 5, 64289 Darmstadt, Germany \\ ${ }^{2}$ GSI Helmholtz-Zentrum für Schwerionenforschung \\ Planckstrasse 1, 64291 Darmstadt, Germany \\ ensinger@ma.tu-darmstadt.de
}

\begin{abstract}
In nature, ion conducting nanopores play a vital role for the function of living cells. They undergo gating processes where they open and close upon an external stimulus, such as the presence of a particular biomolecule, the ligand. When the gating process is observed and is quantitatively measured one can derive data about the presence and the amount of the ligand. Hence, the nanopores can be utilized for specific sensing. However, biological nanopores are embedded in a biological cell membrane that is fragile and unstable with respect to storage and application. The $i N A P O$ project aims at combining robust polymer-based nanopores with protein-based biological nanopores, thus combining the selectivity and sensitivity of the latter with the stability and processability of the first ones. This paper describes the different steps in the fabrication of ion conducting nanopores. It begins with ion irradiation of polymer foils, combined with chemical etching of the ion damage tracks into nanopores. By means of chemical coupling reactions, the nanopore walls are functionalized with particular molecules which react or bioconjugate with the molecules to be analyzed. As an example, a recent result on sensing a physiologically active P-based anion is shown. By means of a complexation reaction with $\mathrm{Zn}$-di(picolyl)amine, the selective measurement of the concentration of the anion pyrophosphate is demonstrated. In the final step of the project, the nanopores will be incorporated into a Lab-on-Chip system for applications in e.g. medical diagnostics and environmental analysis.
\end{abstract}

Keywords: ion track etching, polymer membrane, nanopores, biomimetic sensor, nanosensor, current-potential measurements

\section{Introduction}

Nanopores (or -channels) in biological membranes are highly efficient biomolecular constructions, based on proteins. They control mass transport between the cell interior and its surrounding, including small molecules, water and ions [1]. Despite an often very high transport rate, the nanopores are able to discriminate between different species, i.e. let the one pass and the other one not. This gating process regulates the flow across the membrane and is crucial for the cell functionality. There are several factors that can modulate the transport through the nanopores, depending on the nanopore type, such as mechanical stress, electrical voltage and interaction with specific molecules, the ligands. The latter, for instance, can interact with a specific part of the nanopore and thus open it. Based on the effects of the mentioned gating factors, the observation of the gating process itself can, in turn, be used to monitor the gating factor, such as the presence of the ligand [2]. In other words, if it is observed that the nanopore is in an open state by measuring the mass transport (in case of ion conducting nanopores, it is the ion flow), the ligand must be present. In this way, the nanopore itself acts as a specific sensor [3]. However, there is a drawback for the application of this type of biological sensor: biological proteinbased nanopores/nanochannels have been developed throughout the long-lasting evolution of cells within a biological environment, i.e. within the cell membrane. This renders them fragile, being not suitable for technical environment and application, including aspects such as storage over a longer time period or the integration into electrical devices. It would therefore be desirable to fabricate tailored nanopores from robust materials, such as polymers. This has, indeed, been achieved $[2,4,5]$. However, the selectivity and sensitivity for being able to sense molecular species of interest at very low 
concentrations and in the presence of other similar molecules are still much better for the biological protein-based nanopores in comparison to the polymer-based ones. Hence, the idea of the iNAPO (ion-conducting Nanopore) project is to combine biological protein-based nanopores with polymer-based nanopores. In a further step, they will be integrated into a lab-on-chip system in a micro-device, with a low electrical power consumption and a sensitive readout system, so that eventually a new generation of a micro-tool for (bio)chemical analysis and medical diagnostics is available.

The iNAPO project is based on the expertise of physicists, material scientists, chemists, biologists and electro technicians. It is a bottom-up multi-scale approach. The final iNAPO sensing device is built up from a molecular base of the polymer foil and its modification and functionalization into a micro device in the macro world.

$\left.\begin{array}{|l|l}\hline \text { iNAPO } & \begin{array}{l}\text { lon irradiation } \\ \text { of polymer foil }\end{array}\end{array} \Rightarrow \begin{array}{l}\text { Etching to } \\ \text { nanopore }\end{array} \Rightarrow \begin{array}{l}\text { Chemical } \\ \text { modification }\end{array} \Rightarrow \begin{array}{l}\text { Biological } \\ \text { functionalization }\end{array} \Rightarrow \begin{array}{l}\text { Micro-nano } \\ \text { integration }\end{array}\right\rangle$

Fig. 1: The $i N A P O$ project: Fabrication steps of a micro sensing device for ion conducting nanopores.

The target applications are in the fields of medical diagnostics and environmental analysis, e.g. for tumor marker determination in human body liquids and for measuring toxic components in ground water and drinking water.

In the following, the structure and techniques of the $i N A P O$ project are described and a recent result is given as an example.

\section{Fabrication of polymeric nanopores and their device-integration}

\subsection{Ion irradiation: formation of an ion damage track}

The first step in the fabrication of a nanopore in a polymer foil is the irradiation of the foil with a very highly energetic ion of a heavy element, such as gold. The irradiations are performed at the Universal Linear Accelerator UNILAC of the GSI Helmholtz-Zentrum für Schwerionenforschung (Center for Heavy Ion Research). Gold is vaporized into atoms in the gas phase and ionized into a positively charged state. The gold ions are accelerated in high-frequency electrostatic fields to an extreme high kinetic energy, with a velocity of about $15 \%$ of the speed of light. This extreme energy enables the ion to penetrate a polymer foil in a straight line. For the project, polyester foils are used, namely polycarbonate (PC) and polyethylene terephthalate (PET). These are materials with a wide range of applications, such as optical data storage discs (CDs, DVDs) or the well-known PET bottles. They have several advantages for the iNAPO project: they are well disposable, reasonably prized, and they have an appropriate chemistry both for the nanopore fabrication and for the nanopore modification and functionalization. The foils are being used in a thickness range between 12 and $30 \mu \mathrm{m}$ which is well manageable and processable.

Along its trajectory, each ion projectile induces electronic excitation and ionization processes which finally result in extended electron cascades. As a result of these processes, covalent bonds between the atoms of the polymeric network are cut, leading to severe radiation damage. Small volatile degradation products including hydrogen, hydrocarbons and carbon oxides, migrate out of the foil into vacuum, leaving back a cylindrical so-called ion track, a zone with reduced density and modified chemical reactivity $[7,8]$.

\subsection{Nanopore formation by ion-track etching}

When the irradiated polymer foil is immersed into a solution of an alkaline etchant, the material around the ion track is preferentially etched away, with a rate much larger than the bulk etch rate of undamaged polymer. Thus, each ion track is developed into a cylindrical or a biconical nanopore. For sensing application, however, a conical nanopore is more favorable. Fig. 2 shows schematically the set-up for monitoring the etching process of a single conical nanopore [6,9]. In the two-compartment etch cell, the polymer foil with a single ion track is placed in the cell center, separating the two volumes. One side contains the etchant, a solution of e.g. $9 \mathrm{M} \mathrm{NaOH}$, the other one contains an acidic etch stop, e.g. $1 \mathrm{M}$ formic acid in $1 \mathrm{M} \mathrm{KCl}$ solution. The ion track is dissolved from one side finally converting the track into an open pore. The monitoring of the etching process is done by applying a potential between two gold electrodes inserted in each compartment. Since the polymer is an electrical insulator, no current flows, until the moment of pore breakthrough, when a sudden increase of the current indicates that the nanopore formation process is finished (Fig. 2). The diameter of the small opening of the nanopore, usually in the sub10 nm range, is given by Ohm's law, based upon the known conductivity of the 
etchant solution and the nanopore dimensions. It is defined by the etching time beyond the breakthrough point. Typically, the small opening of the nanopore is around $10 \mathrm{~nm}$ or below, while the large aperture is several $100 \mathrm{~nm}$ wide.
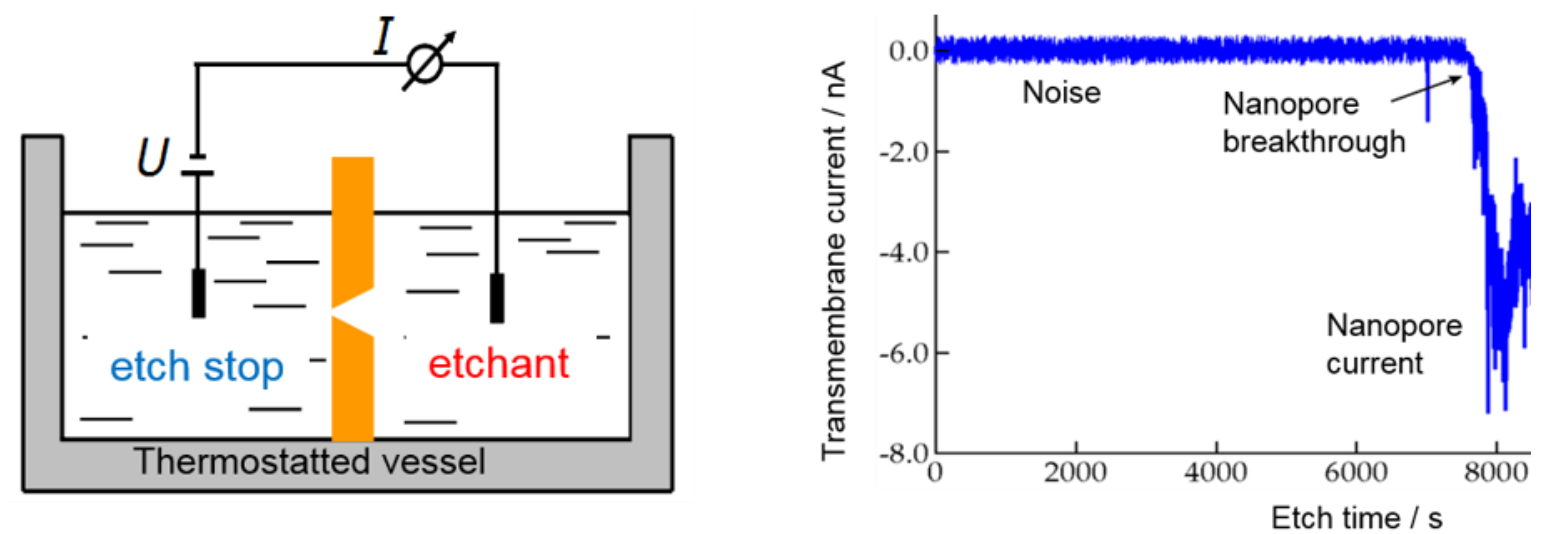

Fig. 2: Schematic presentation of the set-up for etching a conical nanopore into the polymer foil with electrochemical etch control; right hand side: electrolyte current through the nanopore as a function of etch time.

\subsection{Functionalization of nanopores}

The functionalization of single nanopores is done in the following two ways, (i) by attaching specific molecules to the inner pore wall, leading to the so-called first generation nanopores and (ii) by attaching binding proteins as a base for biological protein-based nanopores representing the second-generation nanopores for sensing devices. So far, the first generation functionalization has already been achieved and an example will be shown, while the second generation is in progress with a schematic depicted below for illustration.

Upon chemical etching, the polymer backbone of a polyester is cleaved at the ester groups, leaving back a terminal carboxyl group at the nanopore surface [10]. This is shown in Fig. 3.

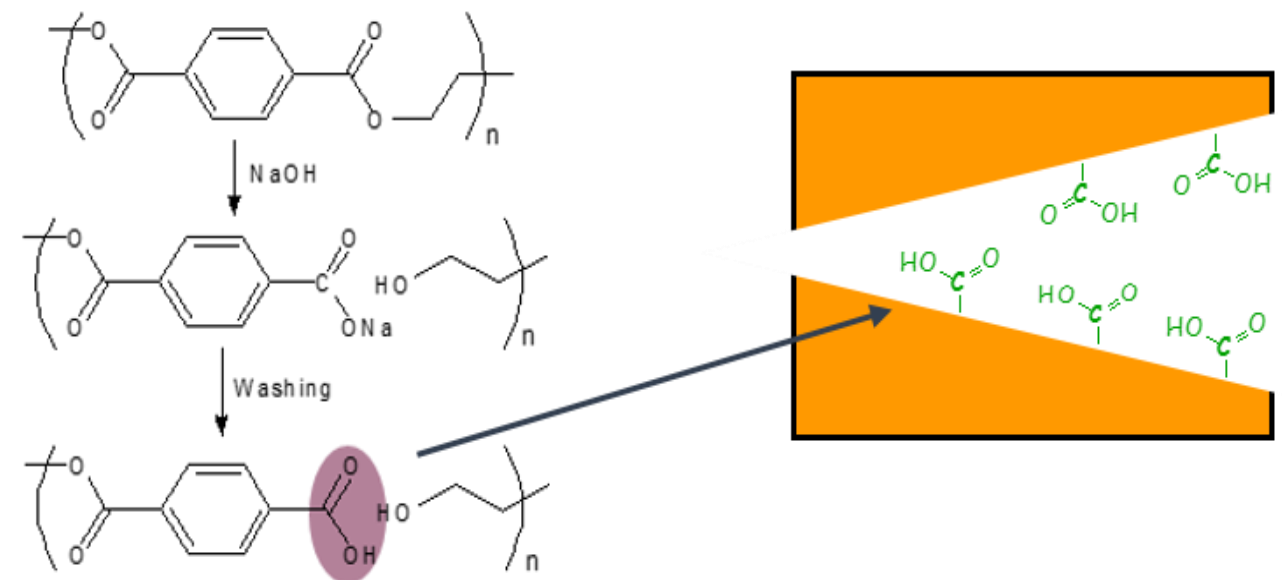

Fig. 3: Alkaline cleavage of polyester backbone leading to terminal carboxyl (-COOH) groups at the nanopore wall.

Carboxyl groups show chemical reactivity to a variety of reactions. Among them is the EDC/PFP coupling reaction that allows for bonding molecules with an amino group. The product is a modified nanopore surface, based on a stable amide bond. The preactivation of the carboxyl group is achieved with dimethylaminopropyl-ethylcarbodiimide (EDC) which reacts further to the penta fluoro phenyl (PFP) ester for a final coupling with an amine. The reaction is shown in detail in Fig. 4. Eventually, the nanopore surface carries a molecular group $R$. This molecule can be the counterpart to another molecule, the one to be analyzed. This analyte molecule specifically reacts with $R$, the receptor. 

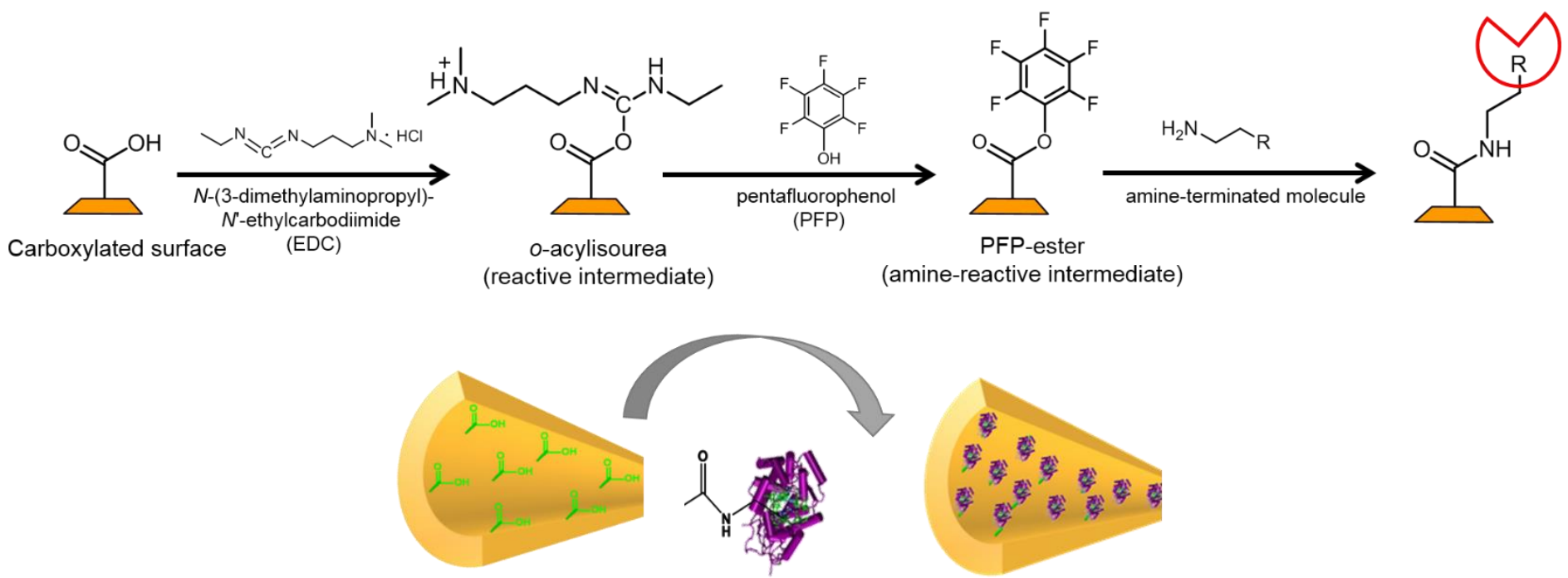

Fig. 4: Coupling reaction for functionalizing the nanopore surface with a reactive group $\boldsymbol{R}$, and schematic presentation of the coupling of a biomolecule onto the nanopore surface via its terminal carboxyl groups.

By means of the EDC/PFP amido reaction, various molecules have been coupled to the nanopore surface, including mannose, a small carbohydrate, or horseradish peroxidase, an enzyme [11,12]. Apart from coupling via covalent bonding, also non-covalent functionalization techniques have been used, such as self-assembly reactions with amphiphilic polymers [13]. The next step in the project will be to insert binding proteins into the nanopores in order to realize biological nanopores. This is schematically shown in Fig. 5.

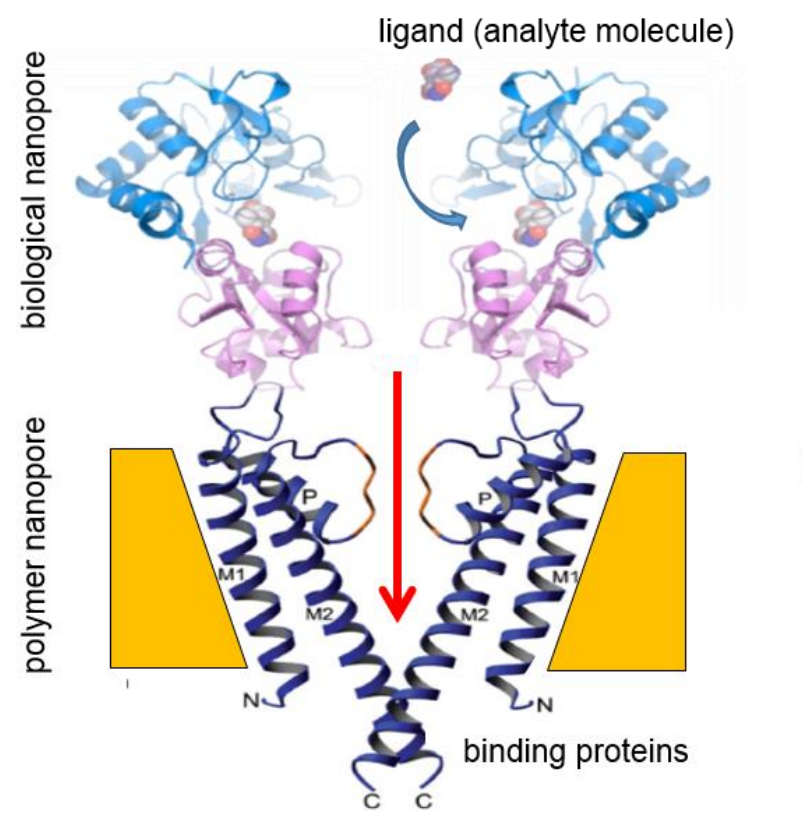

Fig. 5: Schematic cross-section of a polymer nanopore with binding proteins included (dark blue) which are coupled to biological nanopores on top. These are gated by the presence of a specific analyte biomolecule (the ligand, reacting with the receptor) leading to an ion flux (red). 


\subsection{Sensing principle}

Fig. 5 shows already the essential principle of a sensing device. The biomolecule to be analyzed reacts in a specific bioconjugation reaction with the receptor. In case of the biological nanopores, this reaction leads to a change in the nanopore shape, yielding an open nanopore configuration. When the nanopore is open, the ions of the electrolyte can pass and the corresponding electrical current can be measured. This gating is a stochastic event. The state of being open or closed is measured as a function of time. However, this second generation sensing combination of polymeric and biological nanopores is yet to be realized.

An example for the first generation sensor set-up is discussed in the following. As shown above, the wall of the nanopore is functionalized with a biorecognition molecule, the receptor. The measurement setup is the same as shown in Fig. 2. The polymer foil with the nanopore is placed in the center of a two-compartment electrochemical cell. The cell is filled with an aqueous salt solution, such as $0.1 \mathrm{M} \mathrm{KCl}$. This solution exhibits electrical conductivity. When a voltage is applied between the two electrodes, an ionic current flows through the nanopore. When the nanopore surface is electrically neutral, the flow is the same in both directions, irrespective of the polarity of the electrodes. If, however, the surface is charged, the asymmetric shape of the conical nanopores with its potential distribution allows for a larger ion flow in one direction in comparison to the other one. The nanopore becomes a nanofluidic diode, rectifying the current to a certain extent. The resulting current/voltage curve shows a steep line for a positive applied voltage and a flat one under negative voltage, or vice versa, depending on the sign of charge of the nanopore surface.
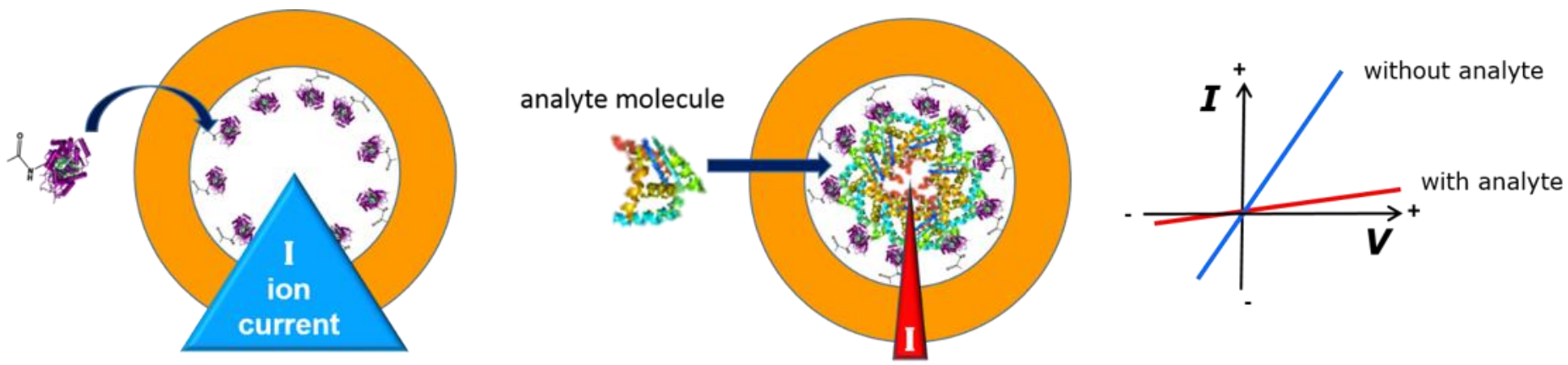

Fig. 6: Schematic view into the nanopore. First, the bioconjugation unit (receptor molecule) is fixed at the nanopore wall. When the analyte molecule is present, it links specifically to the receptor molecule and blocks the nanopore. The pore current $\boldsymbol{I}$ is reduced (from

blue in the open configuration to red in the blocked one). The corresponding current-voltage curves are schematically shown at the

right hand side. The transmembrane current $I$ without analyte molecule is large; in the presence of the analyte it is small.

In biological nanopores, such reactions will be even more specific and sensitive. They will make use of findings from nature. As an example, glutamate recognition by a pentameric ligand-gated ion channel has recently been described [14] and it has been shown which specific domain of the N-methyl-D-aspartate receptor is responsible for activating it by the presence of glycine so that the ion-conducting nanopore opens and ions are able to flow [15].

\subsection{Integration into a micro device}

So far, laboratory set-ups have been used for the measurements. They consist of an electrochemical cell of some 10 to $15 \mathrm{~cm}$ length, connected via cables to a voltage source and a sensitive current meter. The project $i N A P O$ comprises the fabrication of a compact lab-on-chip micro device with the polymer foil embedded in it by means of micro-nanointegration techniques. The polymer foil containing the nanopore is embedded into a microfluidic system with integrated electrodes. The foil is glued between two $200 \mu \mathrm{m}$ thick sheets of the Epoxy-based photoresist SUEX containing three microfluidic channels formed by photolithography. The lower polymer plate is placed on a glass substrate with two evaporated gold electrodes; the upper plate is covered with a glass plate. Details can be found elsewhere [16].

For operation, the microdevice is connected to a power supply and an amperometer that are located in a compact unit where the microdevice is fitted in. The microchannels are filled with electrolyte, and an amperometric reference value is recorded at a given potential (typically a few $100 \mathrm{mV}$ ). Then, the electrolyte with analyte molecules is added and the current response is measured again. In a calibrated system, the concentration of the analyte can be determined quantitatively, if it is within the measurement range of the system. 


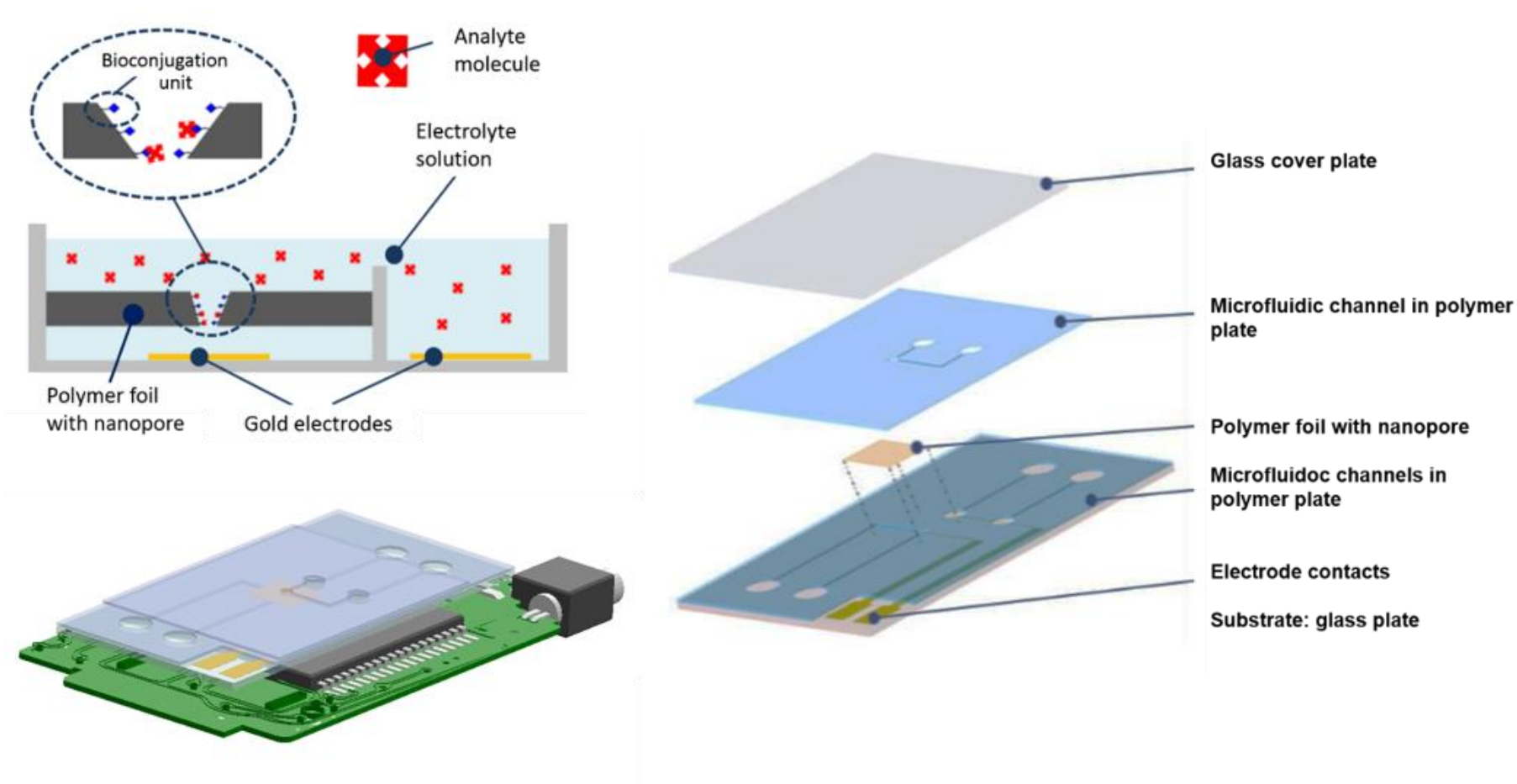

Fig. 7: Schematic presentation of electrochemical microcell with integrated polymer foil containing a single nanopore or an array of a few nanopores; right: setup of the microfluidic lab-on-chip system; adapted from ref. [16]. Bottom left: Microfluidic module on electronic circuit board for insertion into a power supply and read-out unit.

\section{Example for molecule sensing}

The example of a nanopore sensor of the first generation deals with the analysis of pyrophosphate $\left(\mathrm{P}_{2} \mathrm{O}_{7}^{4-}=\mathrm{PP}_{\mathrm{i}}\right)$, an anion which plays an important role in biochemistry [17]. In elevated quantities, it may cause various diseases, such as disordered calcification that may lead to arthritis [18]. Therefore, monitoring is of importance. For a nanopore-based $\mathrm{PP}_{\mathrm{i}}{ }^{-}$ sensor, di(2-picolyl)amine moieties are coupled to the nanopore wall via the above mentioned carbodiimide-based coupling reaction. Zinc ions are added and form a bis $\left(\mathrm{Zn}^{2+}-\mathrm{DPA}\right)$ complex. This complex specifically reacts with $\mathrm{PP}_{\mathrm{i}}$ while there is no reaction with other phosphates such as monohydrogen phosphate $\left(\mathrm{HPO}_{4}{ }^{2-}\right)$, dihydrogen phosphate $\left(\mathrm{H}_{2} \mathrm{PO}_{4}{ }^{-}\right)$, and adenosine mono-, di- and tri-phosphate (AMP, ADP, ATP). Fig. 8 shows schematically the complexation reaction between the zinc-DPA complex and the pyrophosphate and the resulting current/voltage curve.

The ion current through the nanopores is hardly changed when various phosphate ions are added to the electrolyte solution. In contrast, the addition of $\mathrm{PP}_{\mathrm{i}}$ causes a strong reduction of the current at negative voltages, and an increase at the positive voltage branch. These effects are caused by the coupling of $\mathrm{PP}_{\mathrm{i}}$ with its 4 negative charges that influence the cation flow, leading to the diode effect, as mentioned above. Hence, while the presence of other phosphates does merely change the nanopore current, $\mathrm{PP}_{\mathrm{i}}$ can be measured specifically, even down to submicromolar quantities.

Further details are to be found elsewhere [19]. 

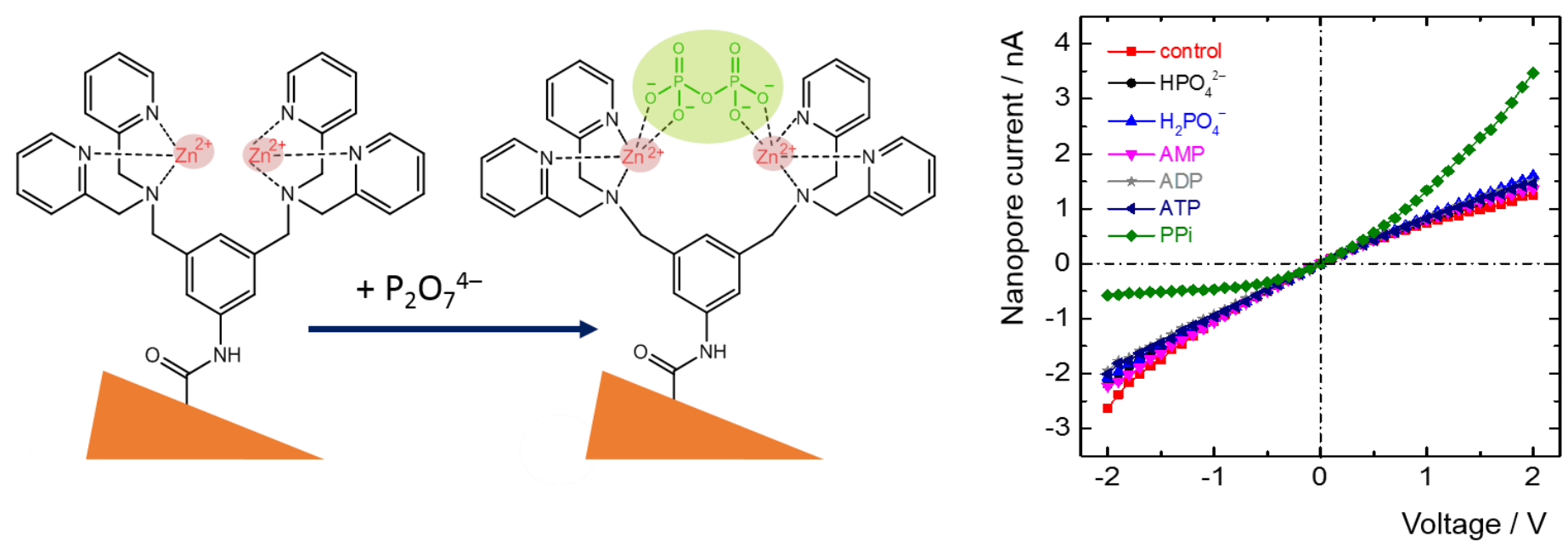

Fig. 8: bis $\left[\mathrm{Zn}^{2+}-\mathrm{DPA}\right]$ complexes coupled to the nanopore surface selectively react with $\mathrm{PP}_{\mathrm{i}}\left(\mathrm{P}_{2} \mathrm{O}_{7}{ }^{4-}\right)$; Right hand side: the nanopore ion currents are significantly changed in the presence of $\mathrm{PP}_{\mathrm{i}}$, but not of other phosphates (monohydrogen and dihydrogen phosphate, and adenosine mono-, di- and tri-phosphate). The concentrations were 1 micromolar; adapted from [19].

Table 1 lists further examples of nanopore-based sensing. The analyte list comprises proteins as well as small molecules and inorganic anions.

Table 1: Nanopore sensing reactions and analyte species.

\begin{tabular}{|l|l|l|l|}
\hline Bioconjugation/ reaction & Bioreceptor / reactand & Analyte & Ref. \\
\hline Streptavidin + Biotin & Biotin & Streptavidin (protein) & {$[20]$} \\
\hline Aptamer + Lysozyme & DNA-Aptamer $($ LyzAp-NH$)$ & Lysozyme (enzyme) & {$[21]$} \\
\hline Mannose + Concanavalin & p-aminophenyl $\alpha$-D-mannopyranoside & Concanavalin A (lectin) & {$[11]$} \\
\hline Enzyme $+\mathrm{H}_{2} \mathrm{O}_{2}$ & Horseradish peroxidase HRP (Enzyme) & Hydrogen peroxide $\mathrm{H}_{2} \mathrm{O}_{2}$ & {$[12]$} \\
\hline F-induced cleavage & Fcn-TBDPS- $\mathrm{NH}_{2}$ & Fluoride $\mathrm{F}^{-}$ & {$[22]$} \\
\hline Ni-Histamine complexation & Nitrilotriacetic-Ni(II) chelate & Histamine & {$[23]$} \\
\hline
\end{tabular}

\section{Conclusion}

The $i N A P O$ project with fabrication of polymeric nanopores, modification and functionalization in combination with protein-based nanochannels is a biomimetic approach that spans the bridge between nature's ion conducting pores and polymer-based pores and comprises their integration in a lab-on-chip device. Not shown here are further aspects, such as accompanying analysis of ionic/fluidic transport in nanopores by Nuclear Magnetic Resonance Spectroscopy and computer simulation. The overall aim is an advanced technology for future applications in medical diagnostics and environmental and process analysis.

\section{Acknowledgement}

This work has been supported in the frame of the LOEWE project $i N A P O$ by the Hessen State Ministry of Higher Education, Research and the Arts. 


\section{References}

[1] B. Hille, Ionic channels of excitable membranes, Sinauer Associates Inc., Sunderland, MA, 2001.

[2] S. M. Iqbal, R. Bashir (Eds.), Nanopores: Sensing and Fundamental Biological Interactions. Springer, Heidelberg, New York, 2011.

[3] H. Bayley, P. S. Cremer, "Stochastic sensors inspired by biology," Nature, vol. 413, pp. 226, 2001.

[4] C. Dekker, "Solid-state nanopores," Nature Nanotechnology, vol. 2, pp. 209, 2007.

[5] M. Tagliazucchi, I. Szleifer, Chemically Modified Nanopores and Nanochannels. Elsevier Science \& Technology, Amsterdam, 2016.

[6] P. Y. Apel, Y. E. Korchev, Z. Siwy, R. Spohr, M. Yoshida, "Diode-like single-ion track membrane prepared by electro-stopping," Nucl. Instrum. Methods Phys. Res. B, vol. 184, pp. 337, 2001.

[7] D. Schauries, M. D. Rodriguez, B. Afra, T. Bierschenk, C. Trautmann, S. Mudie, P. Kluth, "Size characterization of ion tracks in PET and PTFE using SAXS," Nucl. Instr. Meth. Phys. Res. B, vol. 365, pp. 573, 2015.

[8] U. H. Hossain, T. Seidl, W. Ensinger, "Combined in-situ infrared and mass spectrometric analysis of high-energy heavy ion induced degradation of polyvinyl polymers," Polymer Chemistry, vol. 5, pp. 1001, 2014.

[9] Z. Siwy, P. Apel, D. Dobrev, R. Neumann, R. Spohr, C. Trautmann, K. Voss, "Ion transport through asymmetric nanopores prepared by ion track etching," Nucl. Instr. Meth. Phys. Res. B, vol. 208, pp. 143, 2003.

[10] V. B. Gupta and Z. Bashir, Chapter 7, p. 320 in: Stoyko Fakirov (ed.), Handbook of Thermoplastic Polyesters, Wiley-VCH, Weinheim, 2002.

[11] M. Ali, S. Nasir, P. Ramirez, J. Cervera, S. Mafe, W. Ensinger, "Carbohydrate-mediated biomolecular recognition and gating synthetic nanochannels," J. Phys. Chem., vol. C117, pp. 18234, 2013.

[12] M. Ali, M. N. Tahir, Z. Siwy, R. Neumann, W. Tremel, W. Ensinger, "Hydrogen peroxide sensing with horseradish peroxidase-modified polymer single conical nanochannels," Anal. Chem., vol. 83, pp. 1673, 2011.

[13] G. Perez-Mitta, L. Burr, J. S. Tuninetti, C. Trautmann, M. E. Toimil-Molares, O. Azzaroni, "Noncovalent functionalization of solid-state nanopores via self-assembly of amphipols," Nanoscale, vol. 8, pp. 1470, 2016.

[14] T. Lynagh, R. N. Beech, M. J. Lalande, K. Keller, B. A. Cromer, A. J. Wolstenholme, B. Laube, "Molecular basis for convergent evolution of glutamate recognition by pentameric ligand-gated ion channels," Scientific Reports, vol. 2, no. 5, pp. 8558, 2015.

[15] I. Mesic, C. Madry, K. Geider, M. Bernhard, H. Betz, B. Laube, "The N-terminal domain of the GluN3A subunit determines the efficacy of glycine-activated NMDA receptors," Neuropharmacology, vol. 105, pp. 133, 2016.

[16] M. El Khoury, S. Quednau, I. Duznovic, W. Ensinger, H. F. Schlaak, "Integration of Nanochannels for Lab-onChip-Systems," pp. 175, in GMM-Fachbericht 86: Mikro-Nano-Integration, VDE/VDI-Gesellschaft Mikroelektronik Mikrosystem- und Feinwerktechnik (Hrsg), Germany, 2016.

[17] J. K. Heinonen, Biological Role of Inorganic Pyrophosphate. Kluwer Academic Publishers, London, U.K., 2001.

[18] A. E. Timms, Y. Zhang, R. G. G. Russell, M. A. Brown, "Genetic studies of disorders of calcium crystal deposition," Rheumatology, vol. 41, pp. 725, 2002.

[19] M. Ali, I. Ahmed, P. Ramirez, S. Nasir, C. M. Niemeyer, S. Mafe, W. Ensinger, "Label-Free Pyrophosphate Recognition with Functionalized Asymmetric Nanopores," Small, vol. 12, pp. 2014, 2016.

[20] M. Ali, B. Yameen, R. Neumann, W. Ensinger, W. Knoll, O. Azzaroni, "Biosensing and Supramolecular Bioconjugation in Single Conical Polymer Nanochannels. Facile Incorporation of Biorecognition Elements into Nanoconfined Geometries," Journal of the American Chemical Society, vol. 130, pp. 16351, 2008.

[21] M. Ali, S. Nasir, W. Ensinger, "Bioconjugation-induced ionic current rectification in aptamer-modified single cylindrical nanopores," Chemical Communications, vol. 51, pp. 3454, 2015.

[22] M. Ali, I. Ahmed, P. Ramirez, S. Nasir, J. Cervera, C. M. Niemeyer, W. Ensinger, "Fluoride-induced modulation of ionic transport in asymmetric nanopores functionalized with "caged" fluorescein moieties," Nanoscale, vol. 8, pp. $8583,2016$.

[23] M. Ali, P. Ramirez, I. Duznovic, S. Nasir, S. Mafe, W. Ensinger, "Label-free histamine detection with nanofluidic diodes through metal ion displacement mechanism," Colloids and Surfaces B: Biointerfaces, vol. 150, pp. 201, 2017. 